\title{
Crystallography for all - Using the CSD to help promote interdisciplinarity in science
}

\section{Suzanna Ward, Ilaria Gimondi}

\author{
The Cambridge Crystallographic Data Centre (CCDC), Cambridge, United Kingdom; \\ ward@ccdc.cam.ac.uk
}

We are privileged in crystallography that every published crystal structure is shared through established databases and that scientists worldwide can gain new insights from these collections. The Cambridge Crystallographic Data Centre (CCDC) was set up to curate and distribute one of these databases, the Cambridge Structural Database (CSD), a resource containing over one million experimental crystal structures.

As a non-profit organisation sharing data from crystallographers worldwide the CCDC has always had a keen interest in developing material to help others to use structural data to teach both chemical concepts and crystallography. More recently we have realised that we also need to use our position in the scientific community to engage students and researchers to help promote interdisciplinarity in research.

This presentation will highlight some of our efforts to cultivate more interdisciplinarity in science from the establishment of new guidelines, partnerships, links and community initiatives. We will explore recent activities to engage scientists across research areas and ages through our involvement in a variety of schools, workshops and science festivals globally. We will also share our experiences in creating more virtual resources including a new series of CCDC virtual workshops and on-demand training courses through CSD University.

Finally, we will reflect on some of the challenges we have faced, what we have learnt from our experiences and look at what more could be done to increase interdisciplinarity in science.

Keywords: Education, interdisciplinarity, CSD, Data, Training 